\title{
Intermittent positive pressure ventilation by nasal mask: technique and applications
}

\author{
N. Carroll and M.A. Branthwaite \\ Department of Thoracic Medicine, Brompton Hospital, London, UK
}

Received: 13 March 1987; accepted: 6 May 1987

\begin{abstract}
Intermittent positive pressure ventilation delivered non-invasively through a well-fitting nasal mask has been used to control nocturnal hypoventilation in three patients with severe, combined cardiorespiratory and skeletal disease. The advantages of this approach to domiciliary ventilation are described and the requirements of the ventilator are defined.
\end{abstract}

Key words: Sleep - Hypoventilation - Mechanical ventilation

Domiciliary ventilation, usually only at night, is effective treatment for respiratory insufficiency caused by neuromuscular or skeletal disease [1, 2]. Positive pressure ventilation is efficient but requires a permanent tracheostomy, whereas negative pressure methods are cumbersome and sometimes inefficient [3]. A recent abstract [4] reporting five patients with neuromuscular disease treated successfully with positive pressure ventilation delivered through a wellfitting nasal mask (nasal IPPV) prompted use of this method to control nocturnal hypoventilation in patients with severe, combined cardio-pulmonary and skeletal disease. Preliminary experience is presented and the mechanical requirements of the ventilator are discussed.

\section{Case histories}

A 40-year-old women with congenital kyphoscoliosis, ankylosing spondylitis and hypertrophic cardiomyopathy developed worsening cardiorespiratory failure despite treatment with protriptyline [5], intermittent hyperinflation [6], and nocturnal use of nasal continuous positive airway pressure [7]. Uncontrolled hypercapnia (waking $\mathrm{P}_{\mathrm{a}} \mathrm{CO}_{2} 8.0 \mathrm{kP}$; $60 \mathrm{mmHg}$ ), early morning headache and daytime somnolence were attributed to nocturnal hypoventilation (Fig. 1). Nasal IPPV was instituted easily with rapid relief of symptoms, reduction of hypercapnia (waking $\mathrm{P}_{\mathrm{a}} \mathrm{CO}_{2}$ $6.7 \mathrm{kP}_{\mathrm{a}} ; 50 \mathrm{mmHg}$ ), and control of ventilation during sleep (Fig. 2).

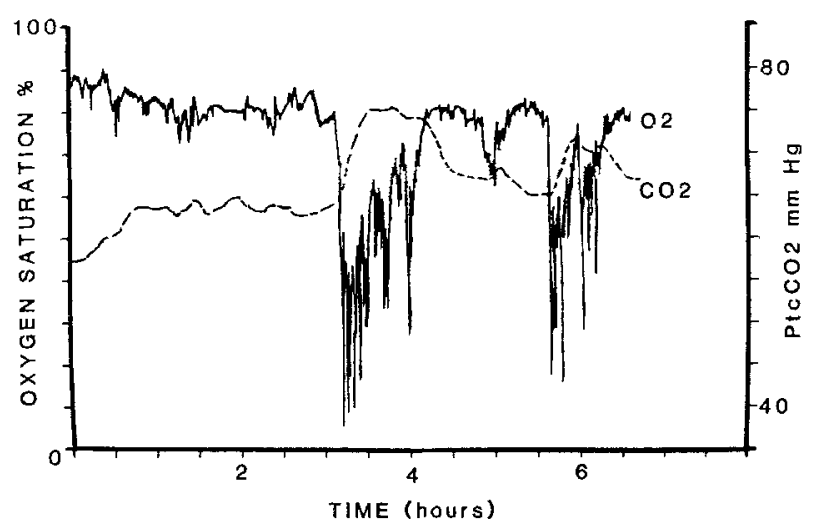

Fig. 1. Overnight recording of oxygen saturation and transcutancous carbon dioxide tension during spontaneous ventilation (Case 1). $\mathrm{PtcCO}_{2}-$ Transcutaneous carbon dioxide tension (approx. $12 \mathrm{mmHg}$ above arterial; $1 \mathrm{kPa}=7.5 \mathrm{mmHg}$ )

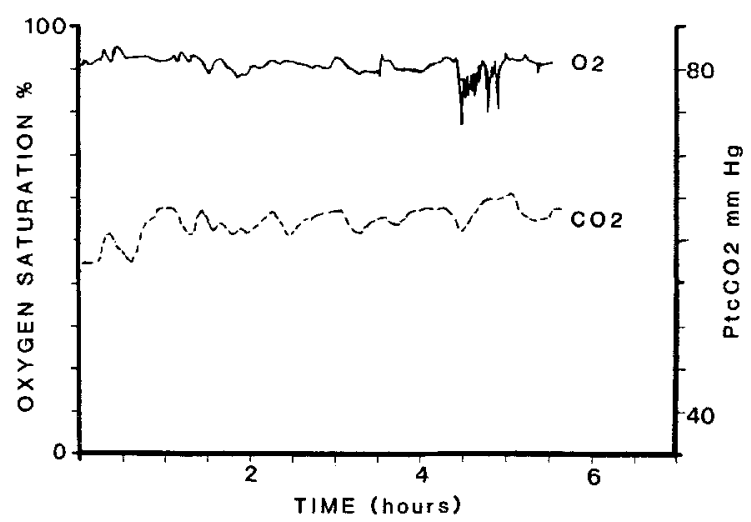

Fig. 2. Overnight recording of oxygen saturation and transcutaneous carbon dioxide tension during intermittent positive pressure ventilation by nasal mask (Case 1 ) 
A 59-year-old man with ankylosing spondylitis (treated by radiotherapy) and bilateral pulmonary fibrosis developed cardiorespiratory failure in 1984. After emergency readmission in 1986, he continued to deteriorate until negative pressure ventilation was instituted with a tank ventilator. Although successful initially, progress was limited by worsening upper airway obstruction during sleep which was incompletely controlled by a nasopharyngeal airway of appropriate length. The change to nasal IPPV was tolerated easily and resulted in complete control of both nocturnal hypoventilation and upper airway obstruction. After 2 months at home, heart failure had not recurred, respiratory failure was well controlled (waking $\mathrm{P}_{\mathrm{a}} \mathrm{O}_{2}$ $8.3 \mathrm{kP}$; $62 \mathrm{mmHg} ; \mathrm{P}_{\mathrm{a}} \mathrm{CO}_{2} 6.6 \mathrm{kP}_{\mathrm{a}} ; 50 \mathrm{mmHg}$ ), and both ventilatory drive (P0.1) and hypercapnic ventilatory response had improved, from 0.164 to $0.41 \mathrm{~cm}$ $\mathrm{H}_{2} \mathrm{O} / \mathrm{mmHg} \mathrm{PCO}_{2}$ and 0.584 to $0.896 \mathrm{l} / \mathrm{min} / \mathrm{mmHg}$ $\mathrm{PCO}_{2}$ respectively.

The third patient developed bilateral pulmonary tuberculosis in childhood. Treatment included left artificial pneumothorax, right plombage, right upper lobectomy and subsequent thoracoplasty. $\mathrm{He}$ first developed cardiac failure at the age of 45 years, and deteriorated in spite of treatment over the next 4 years. Profound nocturnal hypoventilation was documented in 1983 when he was aged 49 . Arterial oxygen saturation was $80 \%$ or less for more than $50 \%$ of total sleep time and added oxygen caused long periods of apnoea and unacceptable hypercapnia. Intermittent obstruction of the upper airway also contributed to nocturnal desaturation, although he was temperate and not obese $(66 \mathrm{~kg})$. Satisfactory control was achieved for 3 years with protriptyline and a cuirass ventilator, but nocturnal hypoxaemia was never eliminated. In 1986 he deteriorated once more with polycythaemia, cor pulmonale, severe airflow limitation (FEV 1/FVC 450/ $900 \mathrm{ml}$ ) and enlarging bilateral bullae. Upper airway obstruction during sleep had become more obtrusive as his cardiorespiratory status deteriorated, until it was impossible to maintain satisfactory gas exchange with a tank ventilator. The transition to nasal IPPV was tolerated well and resulted in complete control of upper airway obstruction, but intermittent hypoventilation persistented if large leaks developed through the mouth during sleep. With added oxygen, and a head halter to support the jaw, it is possible to maintain full saturation during sleep without coincident hypercapnia, allowing the ventilator to control the respiratory cycle when he becomes apnoeic.

\section{Discussion}

The requirements for domiciliary ventilatory support are more stringent if pulmonary disease co-exists with a skeletal abnormality, or if rigidity of the chest wall is of greater importance than weakness of the respiratory muscles. Applying negative pressure to the thoraco-abdominal wall may do no more than assist spontaneous ventilation. This means that hypercapnia is likely if spontaneous ventilatory effort is suppressed by the relief of hypoxaemia, if the patient's efforts do not synchronise with the ventilator and, above all, if there is any tendency to upper airway obstruction [3]. Imbalance between diaphragmatic and upper airway responsiveness is accentuated in respiratory failure when chemosensitivity is reduced [8], and so the frequency of obstructive apnoea is likely to increase. This will be accentuated by negative pressure ventilation but relieved by positive airway pressure. Delivery of IPPV through a well-fitting nasal mask rather than a mouth-piece [9] lessens the magnitude of unwanted gas leaks, eliminates the need for an external humidifier, and may augment respiratory drive by reflex stimulation from the nasal mucosa [10].

Intermittent positive pressure ventilation in the assist/control (triggered) mode eliminates the risk of desynchronisation between patient and machine, provided the trigger is sensitive enough to detect small inspiratory efforts through a potentially leaking airway (the open mouth). The ventilator must also be capable of delivering a greater than normal tidal volume to allow for inefficient pulmonary gas exchange and the possibility of leaks. It must be volume-limited and either time or volume cycled so that it operates regularly despite changes in inflation pressure caused by the variable leak. The response time between initiating the breath and the onset of inflation must be short and the inspiratory flow rate must be amenable to variation over a wide range to suit individual need. Alarms to prevent excessive pressure and to detect unacceptable leaks are also required, and the system must be quiet and reliable for use at home. Few ventilators fulfil this specification and those which do are expensive. The development of less expensive alternatives offers the potential for wider application of this efficient, physiological, and well tolerated technique for controlling nocturnal hypoventilation from almost any cause.

Acknowledgements. NC was supported by a bequest from the estate of the late Mrs. BL Emmerson. Lifecare PLV 100 ventilators were supplied by Thomas Respiratory Systems, London and Respironics nasal masks by Medicaid Ltd., Bognor Regis, West Sussex, UK.

\section{References}

1. Kinnear WJM, Schneerson JM (1985) Assisted ventilation at home: is it worth considering? $\mathrm{Br} J$ Dis Chest 79:313

2. Sawicka $E H$, Loh L, Branthwaite MA (1985) Survival with long term respiratory support. Thorax 40:209 
3. Simonds AK, Branthwaite MA (1985) Efficiency of negative pressure ventilatory equipment. Thorax 40:213

4. Kerby GR, Mayer LS, Floreani A, Pingleton SK (1986) Nocturnal home positive pressure ventilation via nasal mask in patients with hypoventilation from neuromuscular disease. Am Rev Respir Dis 133:A 168

5. Simonds AK, Parker RA, Sawicka EH, Branthwaite MA (1986) Protriptyline for nocturnal hypoventilation in restrictive chest wall disease. Thorax 41:586

6. Simonds AK, Parker RA, Branthwaite MA (1986) Intermittent positive pressure hyperinflation IPPH) in restrictive chest wall disease. Thorax 41:244

7. Sullivan CE, Berthon-Jones M, Issa FG, Evels L (1981) Reversal ob obstructive sleep apnoea by continuous positive airway pressure applied through the nares. Lancet I:862

8. Longobardo GS, Gothe B, Goldman MD, Cherniack NS (1982) Sleep apnea considered as a control system instability. Respir Physiol 50:311

9. Alba A, Khan A, Lee M (1981) Mouth IPPV for sleep. Rehabil Gaz 24:47
10. McNicholas WT, Coffey M, Boyle T, Fitzgerald MX (1986) Stimulant effect of nasal airflow on ventilation during sleep. Thorax $41: 243$

Dr. M. A. Branthwaite

Consultant Physician and Anaesthetist

Brompton Hospital

Fulham Road

London SW3 $6 \mathrm{HP}$

UK

Note added in proof. Since this paper was submitted, two further reports of this technique have appeared:

Ellis ER, Bye PTB, Bruderer JW, Sullivan CE (1987) Treatment of respiratory failure during sleep in patients with neuromuscular disease. Am Rev Respir Dis 135:148

Kerby GR, Mayer LS, Pingleton SK (1987) Nocturnal positive pressure ventilation via nasal mask. Am Rev Respir Dis 135:738 